\title{
Symbiosis and Antagonism
}

\author{
Shogo SASAKI \\ Department of Microbiology, Tokai University School of Medicine, \\ Bohseidai, Isehara, Kanagawa, 259-11
}

(Received for publication, August 8, 1989)

Key words : microbial interaction; symbiosis; antagonism; microbial flora

No organism stands alone: it is a universal truth that none can exist as a singular entity. In the field of microbiology, we deal with units of groups called "clones," but there is no world in which the offspring of any clone exists alone. In other words, the natural world is a continuous turnover of a melting pot of the various clones or species. However, as long as microbiology is to remain a science, it is only natural that we should keep attempting to analyze the phenomena we perceive, in trying to break down the factors into ever simpler forms. In microbiology, we deal with microorganisms in pure culture, making them the object of our studies as "stand-alone" units.

In working with what I will tentatively call "clone A," formed by "organism $\mathrm{x}$," we need to first come to the basic understanding that all organisms comprising this "clone A" are one and the same. The concept of pure culture is based on this basic understanding, even though this is not sacred, and the simple doubt as to whether this is really true does arise. Furthermore, when this "clone A" is mixed with "clone B," comprised by "organism y," belonging to the same species as " $x$ " but different, the problem remains whether the ensuing biological phenomena should be recognized as that appearing as a sum of that inherent to the two, or if there has been an activation of something entirely new, better described as "clone C."

Such correlative relationships are seen in mixed systems wherein differing species exist in the same place and time, and when taken together with other such relationships as with cells and tissue, it becomes doubtful whether it is possible to pursue such group dynamics simply by continuing studies on the individual organism. Hence, it is difficult not to feel the presence of an insurmountable wall standing in the way in terms of the philosophy behind the methodology employed in the research which has been conducted to date with the ultimate goal defined as study of the organs and other such individual entities.

For example, there is the term "host resistance" to infection. Although this is speaking of a relationship established between host and parasite, there are times when it is taken as if it were something which could be expressed in absolute values. 
In other words, there is frequently a lack of appreciation for matters which exist only as correlative relationships, which cannot be expressed as absolute values.

Similarly, there is the concept of "compromised host," which has come to be frequently employed. This too is not an absolute value of the host itself, but is a concept which gains meaning only in the presence of a counterpart.

Also, in discussing the course of infection, unless such problems as adhesion, colonization, invasion, and pathogenecity are each addressed and solved in turn, with analyses of the two correlative systems involved in each case, it is feared that such concepts as "host resistance" and "compromised host" will be taken superficially, without real appreciation of the powers in play.

Another example is the term "opportunistic infection." This indicates the state from infection to onset of disease, as caused by normally non-pathogenic bacteria. This is a concept first expressed by Prof. Rokuzoh Kobayashi in the early 1930s by the words, "expression of pathogenicity by ordinarily non-toxic bacteria"- the pathogenic factor being regarded as toxins at the time. In the same era, Prof. Yuichi Ochi employed the term "spontaneous infection" to describe the same phenomenon. More recently, Prof. Hideo Fukumi has raised the problem of the "infectiousness of bacteria that ordinarily exhibit no pathogenicity." It is believed that although each of these are expressed differently, using terminology and expressions employed in their respective times, they all point to the same concept. However, it is highly unsuitable to include all infections by bacteria with low local pathogenicity as opportunistic infections. Use of the term opportunistic infection should be restricted to describing that occurring between normal flora and host, where prior to the infection there is evidence of longstanding interaction, reaching a certain equilibrium. Opportunistic infections should be defined as that occurring when this equilibrium is broken, and not observing this delineation could lead to unnecessary confusion. Hence, "encounter infections," or infections occurring from what can only be termed chance encounters, where bacteria of low pathogenicity suddenly infect under circumstances totally exceptional to the norm, would not fall into this category in the strict sense. In any case, the term opportunistic infection needs to be employed with caution.

Even more caution is called for with the term "mixed infection." Generally referred to as superinfection or multiple infection, the simple isolation of two species at the same time does not automatically qualify it as a multiple infection by the two species. For example, in considering an infection by two species " $\mathrm{X}$ " and "Y" isolated from the host at the same point in time, there is of course the relationship between "species X" and host, and "species Y" and host, but an equally important factor is the relationship between "species $\mathrm{X}$ and $\mathrm{Y}$," and their subsequent effect on the host, without which there would not be grounds for calling it a mixed infection. Hence, mixed infections can be called as such only when it occurs in a manner defined by a strict set of conditions.

In reviewing these relationships, one sees that they all exist as forms of symbiosis or antagonism. One way of looking at it is as "commensalism," but that is the condition in which both parties have ceased to be antagonistic, but where there is 
a reversion to antagonism once the equilibrium is broken, so that this too can be integrated into the two states of symbiosis and antagonism.

\section{ANTAGONISM-PARTICULARLY WITH REGARD TO HOST FACTORS}

This again takes many forms, but I would like to take the case where the host expels the other as an example.

Figure 1 depicts 3 cases from an infection experiment with germfree mice. In each case, following oral administration of Shigella flexneri $2 a$, a period of approximately 20 days ensues in which about $10^{8}$ viable cells could be recovered from feces. When $E$. coli and enterococcus were introduced upon ascertaining establishment of shigella in the intestinal tract, the count of shigella excreted was drastically reduced, reaching 0 between day 60-80. However, E. coli and enterococcus administered at a later time co-exist, being detected beyond day 180 . Of course, in the controls infected with shigella alone, the shigella was evident beyond day 180. Additionally, when $E$. coli alone was superinfected, the shigella and $E$. coli were found to co-exist, just as the enterococcus co-existed with shigella when it was administered alone.

Furthermore, when $10^{6}$ Shigella flexneri was orally reintroduced at the point when remnants from the first challenge could no longer be detected in feces (around day 160), it was found that they could not colonize at all, being expelled in just a few days.

These results proved to be highly reproducible in further trials involving larger numbers of mice. These facts are important in that they reveal that the first and second encounters between shigella, E. coli and enterococcus culminated in entirely different situations.

When the shigella was replaced by Vibrio cholerae, the combination of E. coli and

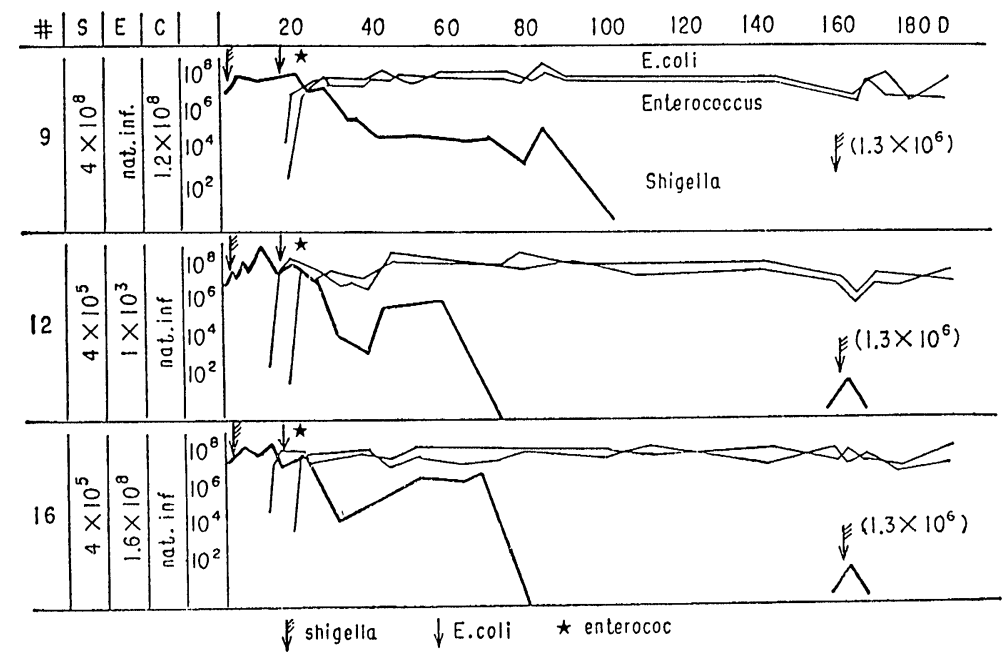

Fig. 1. Antagonism between Shigella flexneri and resident. 
enterococcus proved insufficient for expulsion of the cholera. However, it was proven that addition of Clostridium perfringens to the two led to gradual expulsion of cholera from the system.

These observations are facts seen in mice under experimental conditions, and whether the same holds true in man is not known. Furthermore, as reflected by the considerable difference in expulsion rate brought about by changing the species involved, although the tendency was there in each case, it is clear that these interactions are governed by highly delicate factors (5).

In questioning why the reinfection by shigella did not occur, one is led to the possibility of changes in the host factors. In testing this assumption and for pursuing the interactions between shigella and the host, the effect of changing various host factors were studied in germfree mice.

In Fig. 2, viable counts of shigella isolated from feces are shown on the vertical axis, and days post-oral-infection on the horizontal axis. The course following infection by shigella alone is depicted by line number 1 . In this case, cyclophosphamide, which would inhibit antibody production by the host, was not administered and production of IgA against the shigella is seen. But in the absence of other co-existing species, no free bile acid, or more specifically, 7-ketodeoxycholic acid, is produced. Under these circumstances, the shigella is seen to persist for a long time, being recovered at counts of around $10^{8}$.

Upon introducing cyclophosphamide into this system, IgA production is suppressed as expected, but line 2, depicting the amount of shigella recovered, is very similar to line 1.

By further introducing $E$. coli and enterococcus (noted as strept. in Fig. 2) to the conditions above, there is still no IgA, but bile acid metabolism is promoted and we see the appearance of 7-ketodeoxycholic acid. This state shown by line 3 is

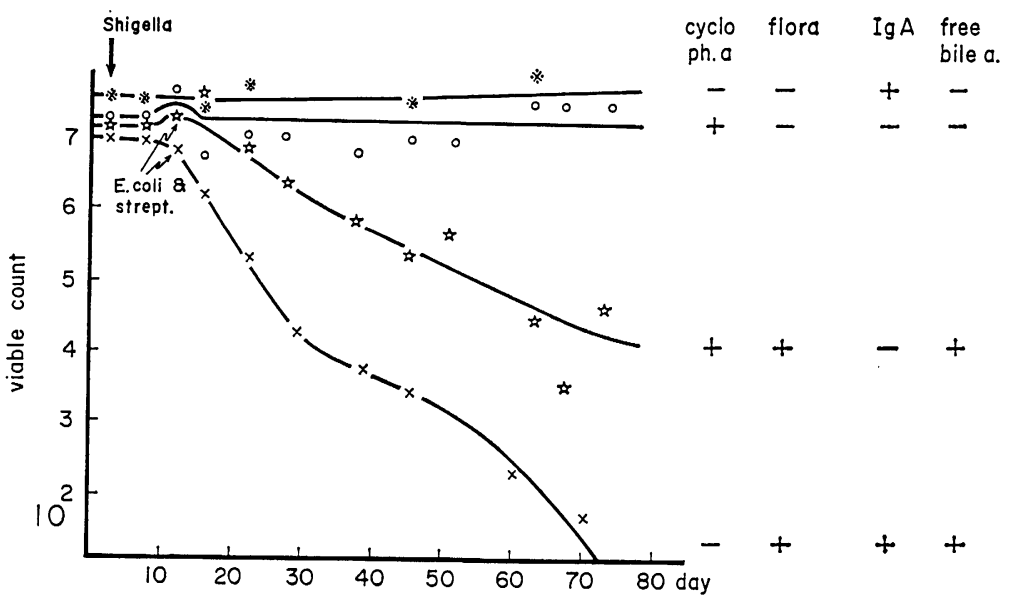

Fig. 2. Influence of cyclophosphamide to persistence of Shigella flexneri. 
clearly different from the two above, and the shigella is reduced to about $10^{4}$ viable cells around day 80 .

Next, in giving $E$. coli and enterococcus without administering cyclophosphamide, both IgA and 7-ketodeoxycholic acid appear, and as shown by line 4, the shigella is no longer detectable around day 70 .

In other words, in addition to the antagonism exhibited by $E$. coli and enterococcus, when the environmental conditions in the intestinal tract are improved by the supply of such host factors as antibody and bile acid, marked expulsion is effected. That is to say that the expulsion is not due solely to the work of the bacteria (5).

\section{ANTAGONISM-PARTICULARLY WITH REGARD TO CYTOTOXIN PRODUCTION}

It is known that Clostridium difficile produces toxin classified as a cytotoxin. In Fig. 3, pH and viable cell count is shown on the left vertical axis, $E h(\mathrm{mV})$ on the right, and days in culture on the horizontal axis. From these results, it can be seen that the cytotoxin is produced as if in response to the viable cell count being maintained at around $10^{7}$ cells, and $\mathrm{pH}$ and $\mathrm{Eh}$ at constant levels.

The effect of adding Peptococcus magnus to this system is depicted by Fig. 4. In short, although the $E h$ in this case was assessed to be lower than that seen in Fig. 3, the Peptococcus magnus reached levels of $10^{9}$, Clostridium difficile to about $10^{7}$, accompanied by consistent cytotoxin production as in the previous experiment.

However, when another anaerobe, Peptostreptococcus parvulus, was substituted for the peptococcus, the $\mathrm{pH}$ and $E h$ were maintained as before, and no change was seen in the number of Clostridium difficile, while cytotoxin production alone was affected, being markedly suppressed (Fig. 5). It is unclear whether this means that the toxin was not produced or that it was broken down and inactivated, but either way, the fact that the toxin titer was reduced is a phenomenon that cannot be denied. Similarly, since this experimental data was collected from in vitro systems, we do not know

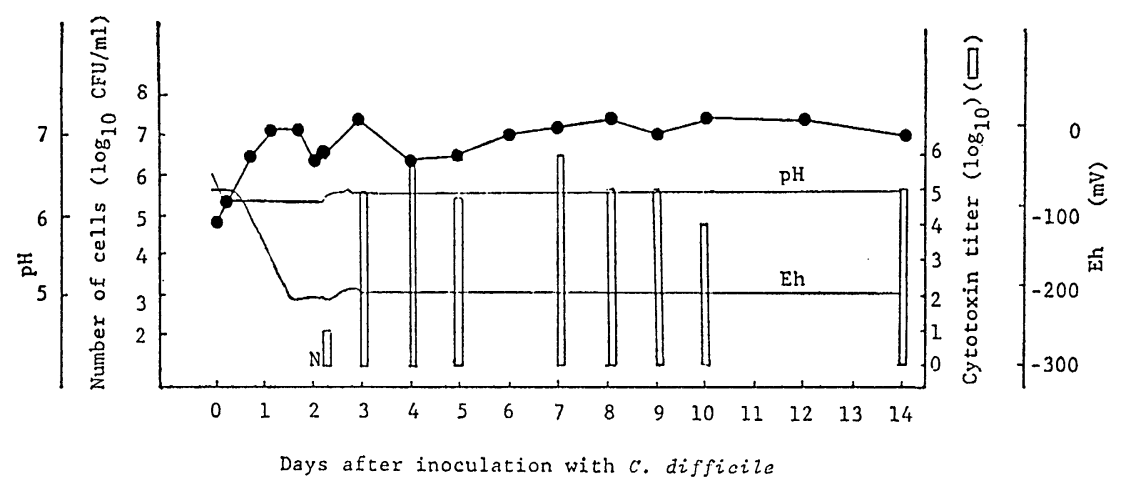

Fig. 3. Growth and cytotoxin production of $C$. difficile in an anaerobic continuous flow culture. number of $C$. difficile cells. $\mathrm{N}$, not detected $(<1)$. 


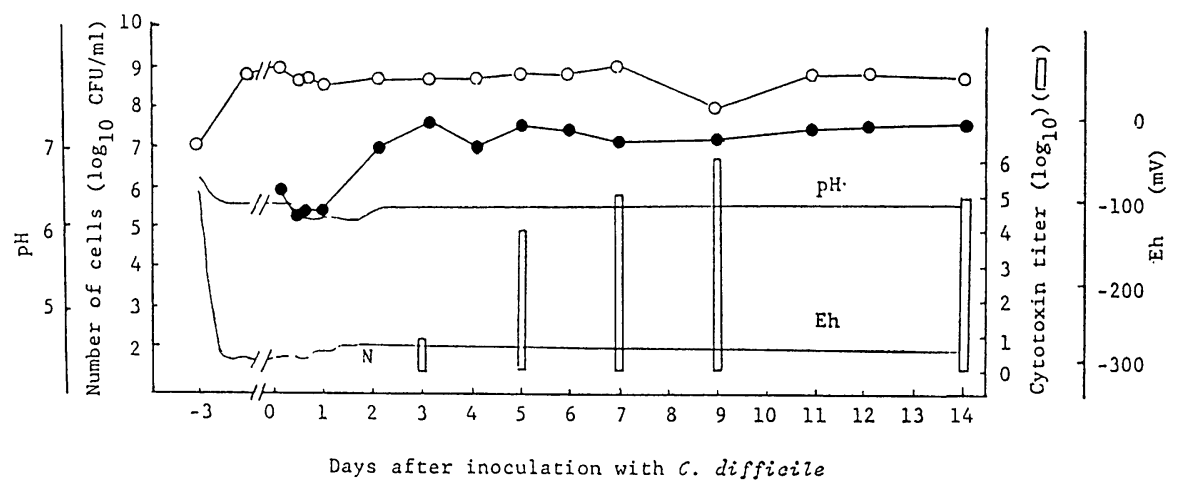

Fig. 4. Growth and toxin production of $C$. difficile in an established anaerobic continuous flow culture of $P$. magnus. $\bigcirc$, number of $P$. magnus cells; $\bullet$, number of $C$. difficile cells. $\mathrm{N}$, not detected $(<1)$.

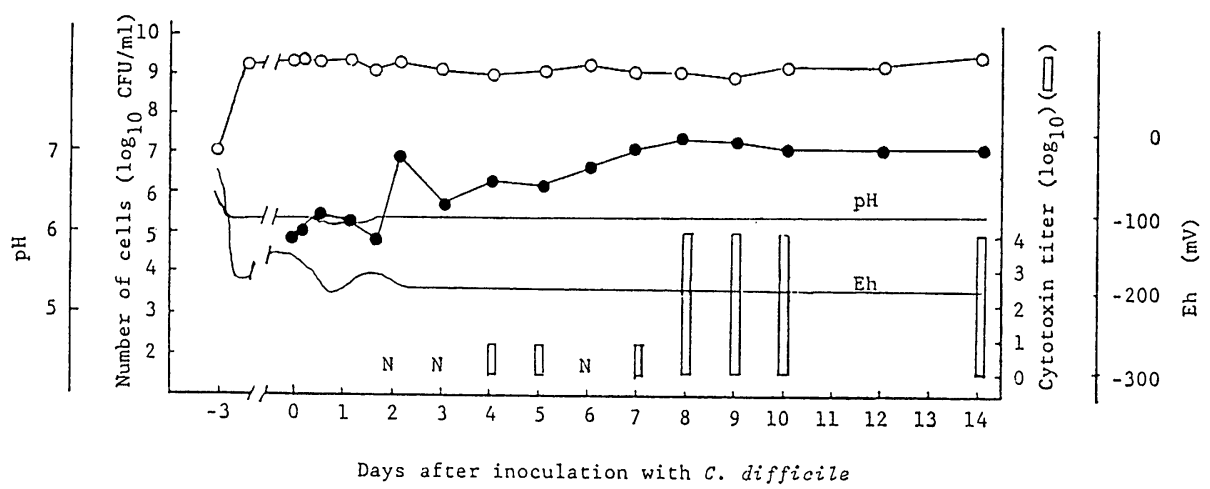

Fig. 5. Growth and toxin production of $C$. difficile in an established anaerobic continuous flow culture of $P$. parvulus. $\bigcirc$, number of $P$. parvulus cells; $\bullet$, number of $C$. difficile cells. $\mathrm{N}$, not detected $(<1)$.

whether the same holds true in vivo, but the point is that these phenomena do exist in the test tube dependent upon the species of bacteria placed therein, portraying one aspect of the antagonistic phenomena(4).

\section{SYMBIOSIS-SPECIFICALLY WITH REGARD TO PATHOGENICITY}

Mice can be infected with mouse cytomegalovirus (MCMV). Injection of $5 \times 10^{6} \mathrm{pfu}$ results in $100 \%$ mortality within a certain time in germfree animals, while the same dose results in $20 \%$ mortality in SPF animals. Reducing the amount of infectious agent to $1 \times 10^{6} \mathrm{pfu}$ brings down the death rates to $64 \%$ and $2 \%$, in germfree and SPF animals, respectively.

Apart from this, injection of 100-500 cfu Klebsiella pneumoniae results in 57\% 
mortality in germfree mice, and $17 \%$ mortality in their SPF counterparts. In this case too, reducing the amount of infection bacteria is reflected proportionately in the death rate (Table 1 ).

Subsequently, a series of experiments was conducted on superinfection by virus and bacteria.

As shown in Fig. 6, when $5 \times 10^{6}$ pfu MCMV was given to germfree mice, followed by $40 \mathrm{cfu}$ klebsiella 7 days later, $100 \%$ of these superinfected animals died on day 5 although all 10 of their controls given single infections were alive on day 12 . Injecting klebsiella on day 10 post-MCMV-infection delayed the onset of mortality somewhat, with 2 survivors. Increasing the interval between MCMV and klebsiella

Table 1. Susceptibility of GF ICR and SPF ICR mice to single infection with MCMV or KP

\begin{tabular}{clcc}
\hline MCMV & Mice & $\begin{array}{c}\text { Number } \\
\text { tested }\end{array}$ & Mortality \\
\hline $5 \times 10^{6} \mathrm{pfu}$ & GF & 5 & $100 \%$ \\
& SPF & 5 & $20 \%$ \\
\hline $1 \times 10^{6} \mathrm{pfu}$ & GF & 39 & $64 \%$ \\
& SPF & 42 & $2 \%$ \\
\hline$* 5 \times 10^{5} \mathrm{pfu}$ & GF & 5 & $0 \%$ \\
& SPF & n.t. & n.t. \\
\hline $1 \times 10^{5} \mathrm{pfu}$ & GF & 5 & $0 \%$ \\
& SPF & n.t. & n.t. \\
\hline
\end{tabular}

\begin{tabular}{cccc}
\hline KP & Mice & $\begin{array}{c}\text { Number } \\
\text { tested }\end{array}$ & Mortality \\
\hline $100-500 \mathrm{cfu}$ & GF & 23 & $57 \%$ \\
& SPF & 6 & $17 \%$ \\
\hline *20-100 cfu & GF & 44 & $41 \%$ \\
& SPF & 26 & $23 \%$
\end{tabular}

* Inoculum size used for double infection, n.t., not tested.
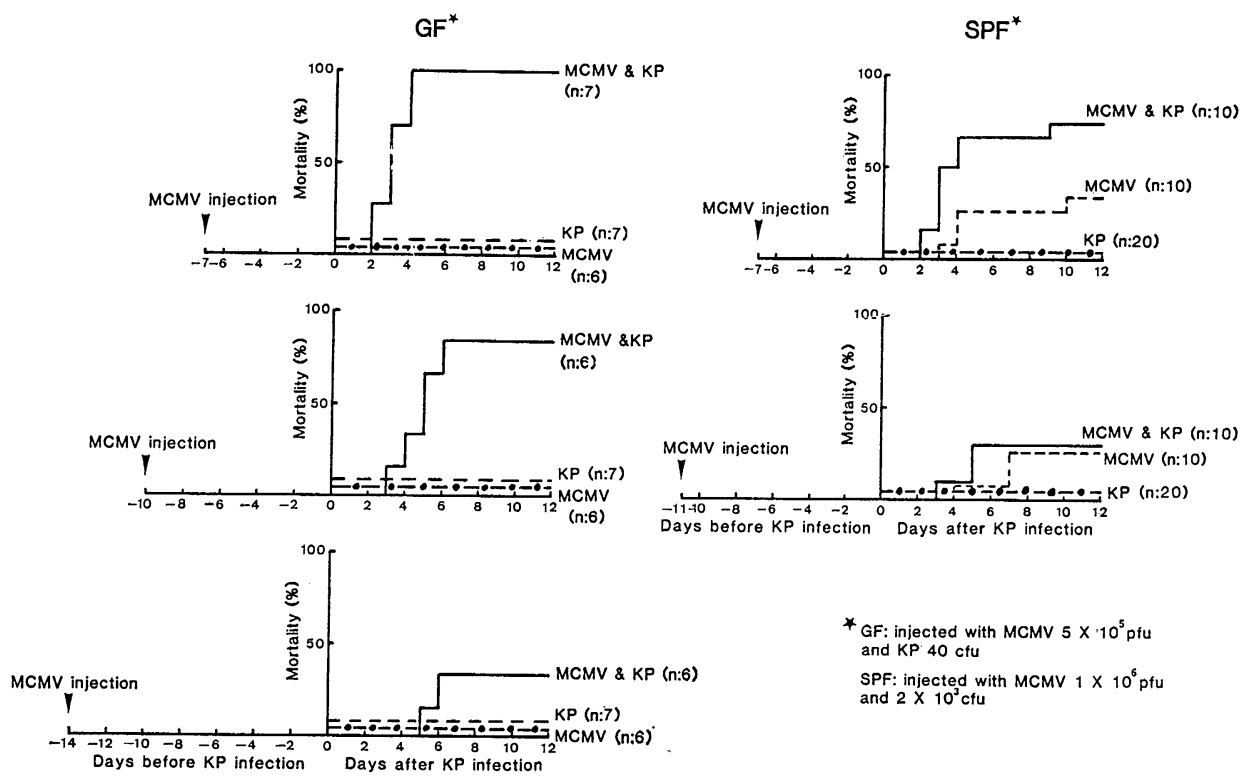

Fig. 6. Mortality of GF or SPF mice infected with $K$. pneumoniae at various times after MCMV infection. 

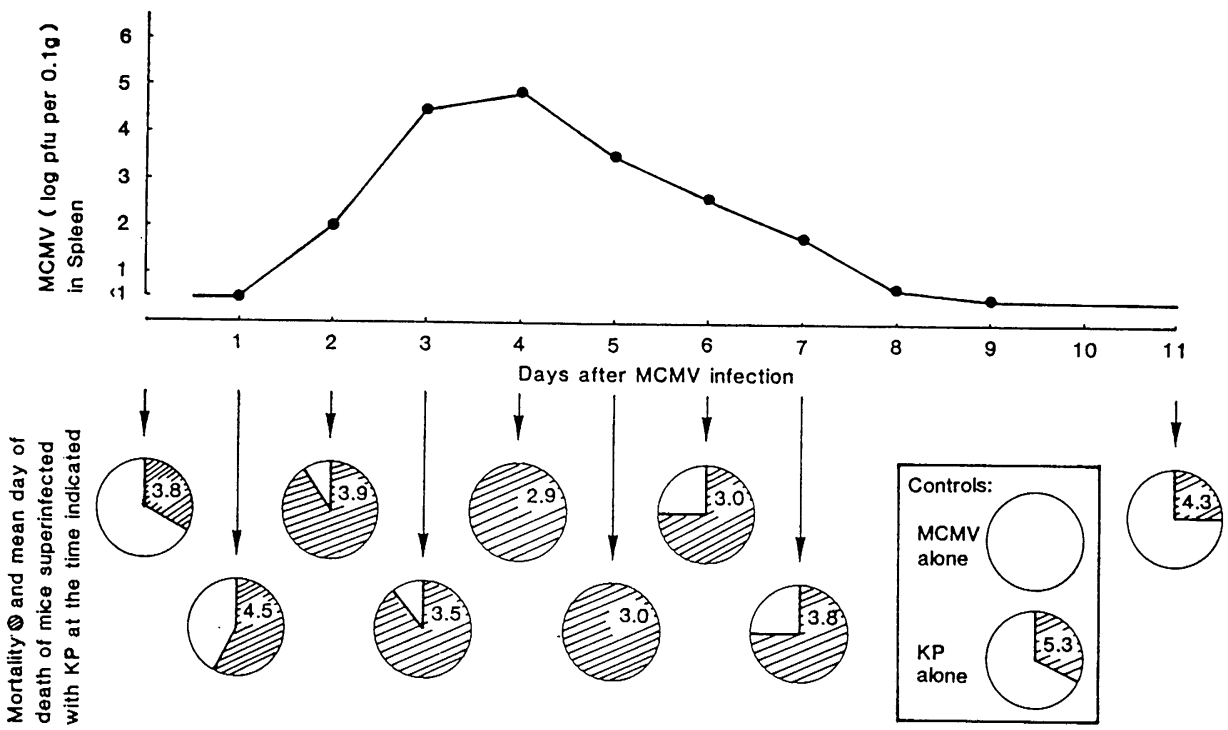

Fig. 7. Relationships between MCMV infection and mortality by $K$. pneumoniae superinfection.

infections even further to 14 days resulted in survival of 6 out of 10 . The same held true for SPF animals.

Graphing the replication of MCMV in the spleen of SPF mice in this experimental series yields the curve shown in the upper half of Fig. 7. Mortality at the respective times is shown in the lower half of the figure: for example, the viral titer in the spleen 1 day post-MCMV-infection is low, and injection of klebsiella at this point results in 50-60\% mortality with death at a mean of 4.6 days. Viral counts reach a maximum at 4 days post-MCMV-infection, and injection of klebsiella at this time results in $100 \%$ mortality at a mean of 2.9 days. In other words, when a counterpart species exists at a time when the viral content is high, a symbiotic relationship is formed between the two, promoting pathogenicity, resulting in antagonism coming into play between the microorganism and host $(1,2)$.

\section{ANTAGONISM AND SYMBIOSIS-PARTICULARLY WITH REGARD TO ANALYSIS OF THE MECHANISMS OF INFECTION}

For using adult mice in oral administration infection experiments, opium has frequently been employed to raise their susceptibility. The mechanism bringing this about is believed to be the suppression of intestinal tract peristalsis by the opium prior to the oral administration of the infectious agent, causing the bacteria introduced to be retained in the intestinal tract for an extended period of time, resulting in an elevated rate of infection. An extreme application of this principle is an experiment method called the De-test. There, the flow of intestinal content is 
stemmed completely by ligating the intestinal tract with sutures, and the presence or absence of infectiousness is tested by introducing the bacteria in question into the tied off section, which is, in effect, an in vivo test tube.

Recently, much attention has been given in pharmacology to the study of opiumoids (opioids), with effects similar to opium, as denoted by its name (3). More than 14 types of opioids have been identified to date; I have heard that one among them is produced by amino acid in casein being split by some species of bacteria. This raised the question of whether bacteria with such capacity might be present in the intestinal tract, producing opioids therein. They would then suppress peristalsis and raise infectiousness, simulating the condition produced by administration of opium.

To examine this possibility, we first tested to see if such bacteria were present in the intestinal tract of normal subjects. The experimental system for this was constructed as follows: In short, the nerves and muscle tissue of guinea pig intestine were removed and placed in medium. Under that condition, the muscle retains a certain myogenic tonus while still being responsive to neurogenic control. When this muscle is stimulated electrically, it contracts further. The mechanism upholding this is the presence of some cells in nerve tissue which produce acetylcholine (ACh), and some which produce opioid, with the greater sensitivity of ACh-producing cells making them subject to weaker electrical stimuli. Therefore, when the stimulation is first transmitted, ACh is produced, which adheres to the receptors on the surface of muscle cells provoking contraction. However, ACh is readily hydrolyzed, being catabolized and inactivated by local ACh-esterase. Additionally, the AChreceptor is blocked by atropine, so that in its presence, ACh is deprived of an area to work in, and its effect does not appear. And from another aspect, it is said that when opioid is produced, it adheres to the ACh-producing cells, inhibiting their function. It is also known that this opioid is neutralized by naloxone.

Going back to the study on bacteria derived from normal subjects, the species isolated were cultured aerobically and anaerobically. The culture supernatants were treated as shown in Table 2, and added to the muscle contraction system previously described.

As shown in Fig. 8, both aerobically and anaerobically cultured preparations had the effect of augmenting muscle contraction, with this effect being observed

Table 2. Preparation of culture supernatant of bacteria employed

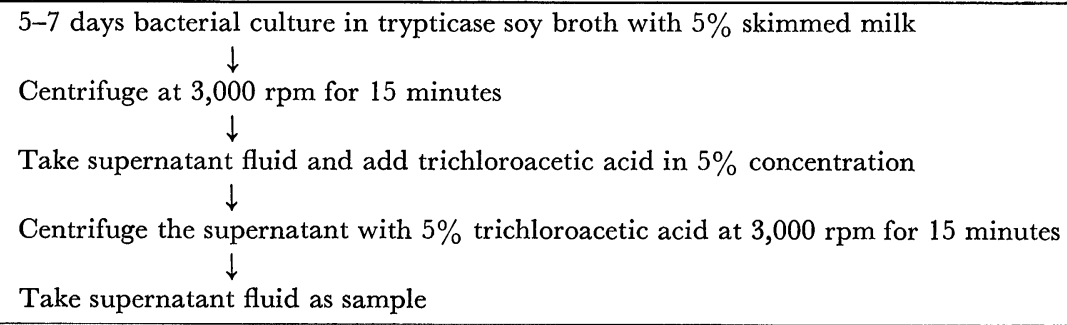




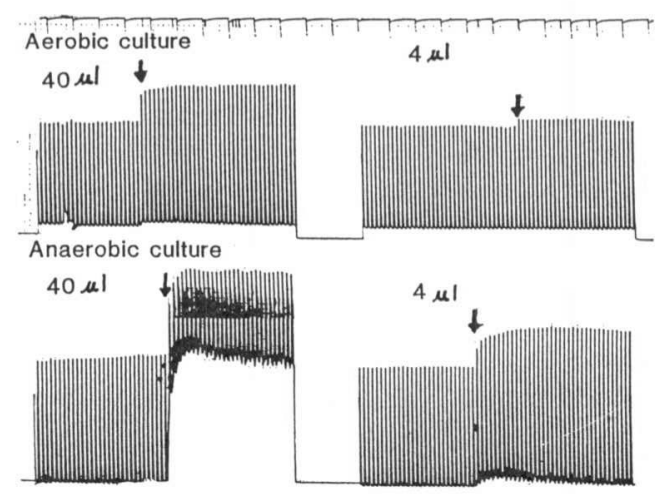

Fig. 8. Effects of culture supernatants of fecal specimens obtained from normal subject on the electrically evoked contractions of the myenteric plexus-longitudinal muscle preparation of guinea pig ileum.

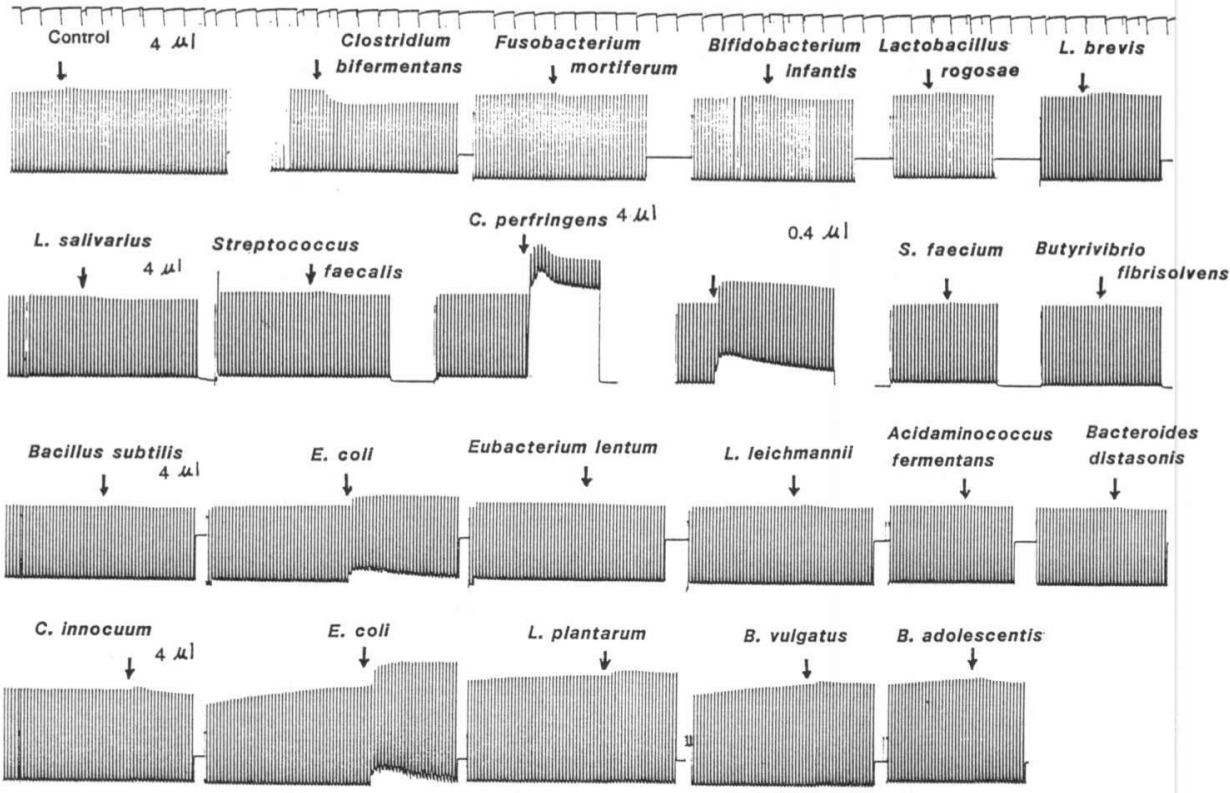

Fig. 9. Effects of culture supernatants of various bacterial species isolated from feces of normal subject on the electrically evoked contractions of the myenteric plexuslongitudinal muscle preparation of guinea pig ileum.

even at $4 \times$ dilution. In other words, it can be seen that both aerobes and anaerobes possess the capacity for strengthening muscle contractions.

Hence, we attempted to isolate the component species as far as possible, and repeated the experiment with each. The results are shown in Fig. 9. It can be seen that there was no difference in the control, and the Clostridium bifermentans 


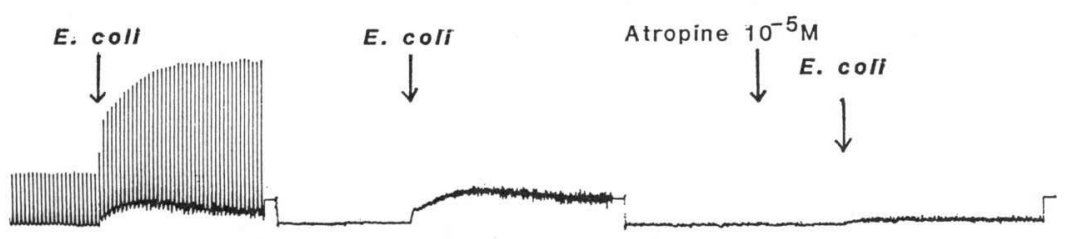

Fig. 10. Antagonism by atropine of culture supernatant of E. coli with increased tension of longitudinal muscle strip of guinea pig ileum.

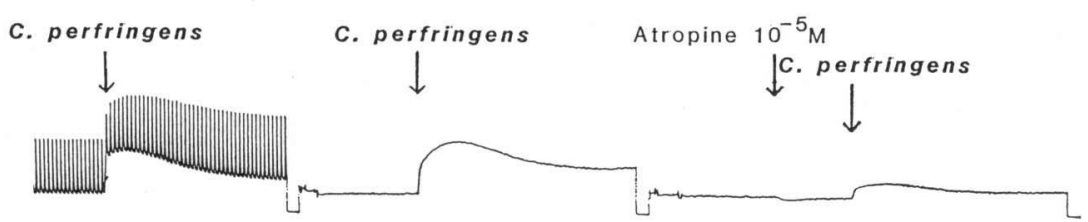

Fig. 11. Antagonism by atropine of culture supernatant of $C$. perfringens with increased tension of longitudinal muscle strip of guinea pig ileum.
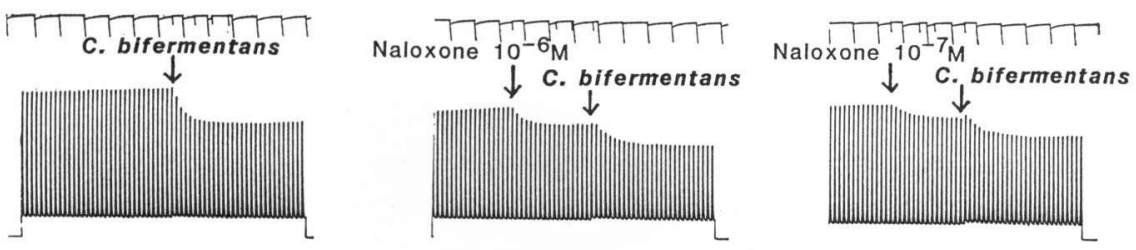

Fig. 12. Non antagonism by naloxone of culture supernatant of C. bifermentans with depression of contractions of guinea pig ileum.

preparation had the effect of suppressing the contractions, resulting in lowering of muscle tonus. Contrary to this, Clostridium perfringens and E. coli preparations augmented the contractions. And with regard to E. coli, diluting the preparation still had a stimulating effect. This effect was cancelled in both E. coli and Clostridium perfringens by the addition of $10^{-5} \mathrm{M}$ atropine (Figs. 10,11). This indicates that rather than the production of opioid, the neutralizing effect of atropine working against $\mathrm{ACh}$ is the more likely mechanism in play. The role of opioid is further denied by the fact that the inhibition of contraction displayed by the Clostridium bifermentans preparation was not neutralized by the addition of $10^{-7} \mathrm{M}$ naloxone (Fig. 12).

What is to be made of the mechanism which stimulates or suppresses the contractions at different times. First of all, the augmenting of contractions occurs when there is extensive $\mathrm{ACh}$ production, little catabolizing enzyme, or lowered levels of atropine or opioid. Conversely, muscle tonus decreases when the reverse condition occurs, or through some entirely different mechanism.

This experiment was not pursued any further from entirely separate considerations, and the question of counterpart species in this equation has been left un- 
answered. Likewise, the relationship between the ACh-and opioid-producing cells among nerve tissue was not addressed. Pursuit of these questions might have facilitated understanding of the initial mechanism coming into play in the onset of diarrhea.

Reviewing these and other such aspects of life, I am of the opinion that organisms do pass through a stage of antagonism, but that they are ultimately proceeding in the direction of symbiosis. In the medical world, there has previously been much work focusing on expulsion. For instance, both surgical methods and chemotherapy were so inclined; but taking blood transfusions as an example, the trend has been for gaining acceptance of cells among different individuals. The trend is even more pronounced in organ transplantations, and work is proceeding on how to go about preventing rejection. In other words, ways are being sought for working out acceptance and symbiosis. Thinking along these lines, perhaps the "anti-biotics" which were developed on the principle of exclusion of the counterparts by sterilization or disinfection will shift to "co-biotics," aiming at co-existence with the counterparts.

To draw an analogy, there is a saying that, when the grass becomes plentiful, there is an increase in rabbits, and subsequently, a greater number of lions, which in turn reduces the number of rabbits, and thence the number of lions, with the grass subsequently growing plentiful again. As can be seen from this, balance in the natural world is an extremely delicate matter.

One cannot help but doubt whether there exists any living entity on earth that can categorically be labeled beneficent or maleficent.

I acknowledge that this holistic approach is not consistent with what has been traditionally accepted as science.

Seeing beauty more in the untouched bloom of flowers in the field than in the cultured form of the beautifully prepared flower arrangement is perhaps an indication of my soul already losing grasp of the scientific spirit.

I would like to thank the President and Chairman for giving me this opportunity to relate my thoughts and some of the data on which they are based.

\section{REFERENCES}

(1) Hashimoto, K. 1986. Experimental analysis of concurrent viral and bacterial infection in the mouse. Tokai J. Exp. Clin. Med. 11 (Suppl.): 59-63.

(2) Hashimoto, K., S. Tazume, K. Umehara, W.C.T. Leung, M. Akiyama, J. Hata, and S. Sasaki. 1987. Increased susceptibility to bacteria of germfree mice infected with murine cytomegalovirus. Gnotobiology and its applications, p. 376-378. In Proceedings of the IXth international symposium on gnotobiology, 1987. Versailles, France.

(3) Oka, T., K. Aoki, M. Kajiwara, and Y. Ishizuka. 1983. Opioid-receptor subtypes. Igakuno Ayumi 127: 378-384.

(4) Ozawa, A., Y. Takahashi, Y. Aiba, K. Yamamoto, K. Furuse, and T. Oka. 1985. Studies on the mechanism of diarrhea induced by antibiotic administration, p. 44-49. In Report of surveillance research committee for malabsorption, Ministry of Welfare of Japan.

(5) Sasaki, S., N. Ohnishi, R. Shimamura, K. Maeda, K. Mizuno, and T. Takahashi. 1973. Significance of intestinal bacterial flora to infection, p. 389-393. In J.B. Heneghan (ed.), Germfree research, biological effect of gnotobiotic environments, Academic Press, New York. 